Published in final edited form as:

Adv Healthc Mater. 2017 December ; 6(24): . doi:10.1002/adhm.201700670.

\title{
Human Periodontal Ligament- and Gingiva-derived Mesenchymal Stem cells Promote Nerve Regeneration when Encapsulated in Alginate/hyaluronic Acid 3D Scaffold
}

\author{
Dr. Sahar Ansari, PhD, \\ Weintraub Center for Reconstructive Biotechnology, Division of Advanced Prosthodontics, School \\ of Dentistry, University of California, Los Angeles, CA 90095 \\ Dr. Ivana M. Diniz, DDS, PhD, \\ School of Dentistry, Universidade de São Paulo, São Paulo, Brazil

\section{Dr. Chider Chen, PhD,} \\ School of Dental Medicine, University of Pennsylvania, 240 South 40th Street Philadelphia, PA \\ 19104
}

\section{Dr. Patricia Sarrion, PhD,}

Weintraub Center for Reconstructive Biotechnology, Division of Advanced Prosthodontics, School of Dentistry, University of California, Los Angeles, CA 90095

\section{Dr. Ali Tamayol, PhD,}

Biomaterials Innovation Research Center, Harvard Medical School, 65 Landsdowne St, Rm 252 Cambridge, MA 02139

\section{Benjamin M. Wu, DDS, PhD [Prof.], and}

Weintraub Center for Reconstructive Biotechnology, Division of Advanced Prosthodontics, School of Dentistry, University of California, Los Angeles, CA 90095

\section{Dr. Alireza Moshaverinia, DDS, MS, PhD}

Weintraub Center for Reconstructive Biotechnology, Division of Advanced Prosthodontics, School of Dentistry, University of California, Los Angeles, CA 90095

\section{Abstract}

Repair or regeneration of damaged nerves is still a challenging clinical task in reconstructive surgeries and regenerative medicine. Here, we demonstrate that periodontal ligament stem cells (PDLSCs) and gingival mesenchymal stem cells (GMSCs) isolated from adult human periodontal and gingival tissues assume neuronal phenotype in vitro and in vivo via a subcutaneous transplantation model in nude mice. PDLSCs and GMSCs were encapsulated in a threedimensional scaffold based on alginate and hyaluronic acid hydrogels capable of sustained release of human nerve growth factor (NGF). We demonstrate that, the elasticity of the hydrogels affected

Correspondence to: Alireza Moshaverinia.

Supporting

Supporting Information is available from the Wiley Online Library or from the author.

The authors declare no potential conflicts of interest with respect to the authorship and/or publication of this article. 
the proliferation and differentiation of encapsulated MSCs within scaffolds. Moreover, we observed that PDLSCs and GMSCs were stained positive for $\beta$ III-tubulin, while exhibiting high levels of gene expression related to neurogenic differentiation ( $\beta$ III-tubulin and GFAP) via qPCR. Western blot analysis showed the importance of the elasticity of the matrix and the presence of NGF in the neurogenic differentiation of encapsulated MSCs. In vivo, immunofluorescence staining for neurogenic specific protein markers confirmed islands of dense positively stained structures inside transplanted hydrogels. To our knowledge, this study is the first demonstration of the application of PDLSCs and GMSCs as promising cell therapy candidates for nerve regeneration.

\section{Graphical abstract}
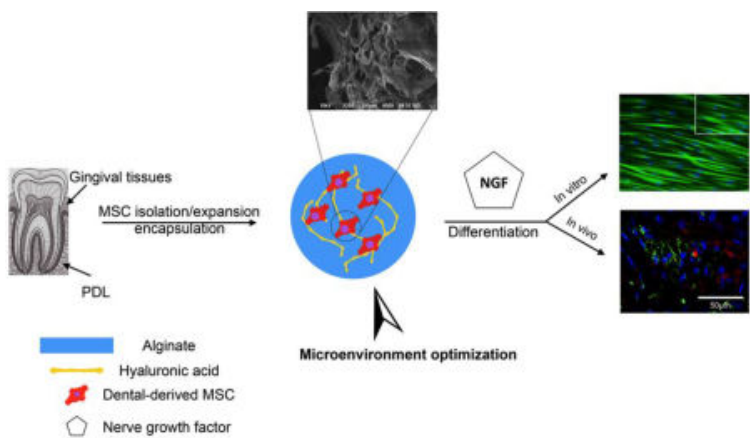

A 3D hydrogel delivery system is fabricated based on alginate and hyaluronic acid hydrogels, with optimized elasticity. Periodontal $\underline{\text { Ligament }} \underline{\mathrm{S}} \mathrm{em} \underline{\mathrm{C}} \mathrm{ells}$ (PDLSCs) and Gingival Mesenchymal $\underline{\text { Stem }}$ Cells (GMSCs) are encapsulated in the engineered scaffold and it is demonstrated that proliferation and differentiation of encapsulated MSCs toward neurogenic tissues is regulated by matrix elasticity and the presence of a suitable signaling molecules. It is shown that these MSCs encapsulated in a 3D injectable cell delivery scaffold might be promising candidates for cellularbased treatment for nerve tissue engineering.

\section{Keywords}

Alginate; Hyaluronic acid; Nerve regeneration; Dental-derived mesenchymal stem cells

\section{Introduction}

One of the most challenging obstacles in reconstructive surgery and regenerative medicine is the regeneration and the repair of damaged nerves. ${ }^{[1,2]}$ The inability to regenerate the lost nerve tissue has detrimental consequences such as permanent loss of function or posttraumatic morbidity. ${ }^{[1,2]}$ It is well known that the nervous system, both central and peripheral, has a limited self-repair/regeneration potential. One of the common treatment modalities for regeneration of nerve tissues is nerve autografting, in which a nerve graft is harvested from a functionally less important nerve. ${ }^{[1-3]}$ However, this treatment modality has many drawbacks, such as donor site morbidity, the possibility of neuroma formation, scarring, and the expense of the surgical procedure. ${ }^{[1-3]}$ Cell replacement therapies have shown promise as an alternative modality of treatment for nerve regeneration. ${ }^{[4-6]}$ Studies 
have confirmed that embryonic stem cells (ESCs) are capable of differentiation toward most neuronal subtypes as a promising cell source for nerve regeneration treatments. ${ }^{[7,8]}$ As well as induced pluripotent stem (iPS) cells, neural stem cells (NSCs) have also revealed potential for use in nerve regeneration therapies. ${ }^{[9,10]}$ Studies have confirmed the presence of NSCs throughout life in both humans and animals. Currently, cell therapies are the best alternative to a nerve graft. ${ }^{[9-11]}$ However, in a situation where the gap at the defect site is too long, these approaches may not lead to favorable outcomes. ${ }^{[10,11]}$ Additionally, NSCs are not always easy to access or isolate. Alternatively, mesenchymal stem cells (MSCs) can be considered as a promising source of stem cells for nerve regeneration applications. ${ }^{[12-14]}$ MSCs are multipotent cells that, depending on the received signals from microenvironment, can be differentiated into osteogenic, adipogenic, chondrogenic, neurogenic and other lineages. ${ }^{[15-17]}$ Several studies have shown that MSCs have the potential to differentiate into functional neural cells, including astrocytes, neurons, and oligodendrocytes. ${ }^{[18-20]}$ Several of these reports on neurogenic differentiation have concentrated on bone marrow MSCs (hBMMSCs). Though, hBMMSCs are identified to have a low and unpredictable differentiation capacity relative to certain other types of MSCs. ${ }^{[13,14,18-20]}$ Therefore, it seems essential to find an optimal cell source for nerve regeneration and repair applications.

MSCs are found in a variety of post-natal structures, including many in the orofacial region. ${ }^{[21-26]}$ Numerous kinds of dental-derived MSCs have been extracted and characterized, such as: periodontal ligament stem cells (PDLSCs) and more recently, gingival mesenchymal stem cells (GMSCs). ${ }^{[21-26]}$ These two categories of MSCs are easily available in the oral cavity; indeed, they can also be found in dental clinics tissue waste. ${ }^{[27-30]}$ Furthermore, our group and others have confirmed their multilineage differentiation capabilities. ${ }^{\text {[27-30] }}$ Therefore, PDLSCs and GMSCs might be unique and promising cell types for nerve regenerative therapies.

It's very accepted that the microenvironment has a fundamental role in determining stem cell fate. ${ }^{[31,32]}$ Signals that cells receive from signaling molecules or from the extracellular matrix (ECM) direct the differentiation of MSCs toward the desired lineage. ${ }^{[33,34]}$ Considering the three dimensional (3D) organized assembly of the central nervous system (CNS), a 3D biomaterial seems to be necessary to provide a suitable microenvironment for differentiation of encapsulated MSCs toward neurogenic tissues. ${ }^{[35]}$ Several types of biomaterials have been used as 3D delivery vehicles for MSCs. Among these biomaterials, alginate hydrogel is one of the popular choices. ${ }^{[36,37]}$ Alginate is a natural heteropolysaccharide derived from algae. ${ }^{[38,39]}$ Sodium alginate ionically crosslinks by divalent cations (e.g. $\mathrm{Ca}^{2+}$ ). Alginate has found a wide variety of applications in bioengineering based on its favorable features, including biocompatibility, non-toxicity, and gentle gelation. ${ }^{[38,39]}$

Alginate has been used successfully to direct differentiation of encapsulated MSCs or iPCSs toward neural lineage phenotypes. ${ }^{[35,40,41]}$ However, it is well known that alginate hydrogel without any modification does not provide suitable cell adhesion. ${ }^{[38,39]}$ Hence, to mimic the cell-interactive characteristics of the ECM, cell binding motifs (e.g., RGD, an arginineglycine-aspartic acid tripeptide), have been coupled to it. ${ }^{[42,43]}$ Another natural hydrogel biomaterial that is a promising matrix for nerve regeneration is hyaluronic acid (HA), a 
glycosaminoglycan that is one of the important constituents of the ECM of the developing CNS and neural stem cell niche. ${ }^{[45]}$ Studies have confirmed the importance of this hydrogel in promoting cell survival, migration, and differentiation for nerve regeneration. ${ }^{44-46}$

For successful tissue regeneration applications, the hydrogel biomaterial should possess biomechanical properties close to those of the natural ECM to provide an optimal environment for the growth and differentiation of encapsulated cells toward the desired phenotype. To develop a suitable microenvironment for nerve regeneration based on PDLSCs or GMSCs, we planned to engineer an RGD-conjugated alginate and hyaluronic acid (HA)-based hybrid hydrogel scaffold with a low elastic modulus to direct neurogenic differentiation of the encapsulated PDLSCs and GMSCs.

\section{Results}

\subsection{Viability of encapsulated cells in HA/alginate hydrogels}

In the current study, PDLSCs and GMSCs were isolated from periodontal or gingival tissues of patients with healthy periodontium. The isolated MSCs were expanded in vitro, encapsulated in one of the fabricated Hyaluronic acid (HA)/alginate hydrogels and utilized for neurogenic differentiation assays in vitro and in vivo. Human BMMSCs were used as the positive control, while cell-free hydrogels were used as the negative one. Subsequently, the viability of the encapsulated MSCs and their neurogenic differentiation capacity were analyzed. Our flow cytometric analysis data confirmed the stemness of these MSCs by expression of specific MSC surface markers such as: CD73, CD105, and CD146 (not shown), while, both of these cells failed to express hematopoietic lineage markers including CD34 or CD45. MSCs were encapsulated in hydrogel scaffolds at a cell density of $1 \times 10^{6}$ cells $/ \mathrm{mL}$ and the viability of MSCs was measured at three time intervals: after 1,7 and 14 days of culturing. Our live/dead staining results exhibited high in vitro viability for PDLSCs, GMSCs, and hBMMSCs encapsulated in fabricated hydrogels at different time intervals of culturing in regular culture media (Figure 1a and 1b).

\subsection{Characterization of fabricated hydrogels and analysis of cell proliferation}

HA content affects properties of hydrogels including their elastic modulus and porosity. It is well known that the elastic modulus of the matrix plays a role in directing the fate of encapsulated cells. ${ }^{[53]}$ Therefore, we fabricated and tested three different hydrogels with different alginate/HA ratios (4:1, 2:1, and 1:1 w/w). Subsequently, the mechanical properties of the fabricated hydrogels were characterized. The data exhibited in Figure 2a show the elastic modulus of the fabricated hydrogels with different compositions. The formulation with the highest concentration of HA (Alg/HA: 1:1) exhibited the lowest elastic modulus (5 $\mathrm{KPa}$ ) of the four tested hydrogels. Alginate hydrogel alone showed the greatest elasticity (18 $\mathrm{KPa}$ ). Additionally, the morphological structure of the fabricated hydrogels was analyzed using SEM and our data confirmed a porous morphology (Figure 2b) with pore size ranging from $45 \mu \mathrm{m}$ to $89 \mu \mathrm{m}$. By increasing the ratio of hyaluronic acid in the hydrogel mixture slight increase in the porosity was observed (Figure 2c). 
Moreover, we aimed to assess the influence of the elasticity of the 3D fabricated hydrogel on the proliferation of encapsulated MSCs. All the encapsulated MSCs showed a significant increase in the proliferation rate (cell number) after one week of culturing (Figure 2d).

It is well known that the proliferation rates of cells cultured in 3D and 2D can be different. Studies have confirmed that decreased proliferation rate in $3 \mathrm{D}$ cultures in comparison to $2 \mathrm{D}$ cultures for variety of cell lines. ${ }^{[61]}$ In contrast, some other cell lines have exhibited an opposite trend showing increased proliferation rates in $3 \mathrm{D}$ cultures than $2 \mathrm{D}$ ones. ${ }^{[61]}$ Interestingly, the proliferation rate of GMSCs increased with a decrease in the elasticity of the fabricated hydrogels (Figure 2e). However, the proliferation rates of PDLSCs and hBMMSCs seemed not to be significantly affected by changing the elasticity of the encapsulation hydrogel (Figure 2f). The amount of increase in the proliferation rate for MSCs cultured in a 2D monolayer without hydrogel after one week of culture was found to be slower (data not shown here) than that of encapsulated MSCs, suggesting that scaffold chemical composition and biomechanical properties influence cell proliferation.

In the next step, the release profile of the incorporated $\beta$-NGF from the fabricated alginate/HA hydrogels was characterized. Figure 3 a shows the cumulative release of $\beta$-NGF from the HA/alginate hydrogel scaffolds. The results confirmed continued release of $\beta$-NGF for up to 14 days. An increase in growth factor release was observed from the alginate/ hyaluronic acid hydrogel with the highest concentration of HA; however, no significant difference was observed in the release of the growth factor from the four tested scaffolds for up to two weeks ( $p$ value $>0.05$ ). Moreover, immunofluorescent staining with the anti- $\beta$ NGF antibody showed that less $\beta$-NGF was retained in Alg/HA (1:1) hydrogel than alginate hydrogel without HA after 14 days (Figure $3 b$ and $3 c$ ). Results of the swelling kinetics study are presented in Figure 3d. It is well known that HA is a highly hydrophilic structure that can swell easily. ${ }^{[60]}$ Our data showed that by increasing the ratio of HA in the hydrogel mixture, a decrease in swelling ratio was observed which is well related to the balance of the ionic strengths between the polyanions and polycations in the alginate/HA hydrogel mixture. [60]

\subsection{In vitro differentiation of dental-derived MSCs}

MSCs cultured in a 2D neurogenic inductive medium after $72 \mathrm{~h}$ of incubation exhibited long and multibranched-liked structures, while the MSCs cultured in regular media failed to exhibit the abovementioned morphology (Supplementary Figure S1). After two weeks of culturing PDLSCs and GMSCs in vitro, positive BIII-tubulin immuno-staining confirmed neurogenic differentiation. Interestingly, PDLSCs and GMSCs exhibited higher levels of expression of $\beta$-tubulin III than hBMMSCs (Figure 4a). However, MSCs cultured in the absence of $\beta$-NGF failed to express any positive staining. We also encapsulated MSCs in different fabricated hydrogels in the presence of $\beta$-NGF and analyzed their neurodifferentiation through immunofluorescence. MSCs encapsulated in alginate/HA with a 1:1 ratio showed higher expression levels of $\beta$ III-tubulin compared to MSCs encapsulated in hydrogels with lower concentrations of $\mathrm{HA}(\mathrm{Alg} / \mathrm{HA}: 4: 1$ and 2:1) or alginate alone $(\mathrm{p}<0.05)$. Additionally, PDLSCs showed statistically greater amounts $(\mathrm{p}<0.05)$ of $\beta$ IIItubulin staining in comparison to GMSCs or hBMMSCs (Figure $4 \mathrm{~b}$ and $4 \mathrm{c}$ ). No statistically 
significant difference was observed between expression levels of GMSCs and hBMMSCs $(\mathrm{P}<0.05)$.

Gene expression analysis was performed to evaluate the molecular mechanism of neurodifferentiation of encapsulated MSCs. $\beta$ III-tubulin and GFAP expression levels were analyzed (Figure 5a). Our quantitative PCR analysis showed that PDLSCs and GMSCs both abundantly expressed $\beta$ III-tubulin and GFAP two weeks after in vitro differentiation (Figure 5a). However, our quantitative analysis of gene expression demonstrated that PDLSCs showed higher expression levels of genes associated with neurogenic differentiation than GMSCs or hBMMSCs ( $\mathrm{p}$ < 0.05) (Figure 5a). Moreover, encapsulated MSCs in hydrogels with the lowest modulus of elasticity (Alg/HA 1:1) showed statistically higher ( $\mathrm{p}<0.05$ ) levels of neurogenic-related gene expression than MSCs encapsulated in a matrix with higher elasticity. These data indicate that the best condition for neural differentiation is an alginate/HA matrix at a ratio of $1: 1 \mathrm{w} / \mathrm{w}$ in the presence of $\beta$-NGF. Additionally, Western blot analysis results were in agreement with PCR findings showing that PDLSCs showed higher expression levels of GFAP and $\beta$ III-tubulin compared to GMSCs or hBMMSCs (Figure 5b). Our Western blotting data showed that PDLSCs encapsulated in a softer scaffold (alginate/HA with a 1:1 w/w ratio) exhibited a higher expression level of $\beta$ IIItubulin than PDLSCs encapsulated in stiffer scaffolds (HA/alginate at 2:1 or 4:1 w/w ratios) (Figure 5c), suggesting that the hydrogel elasticity can influence MSC differentiation towards the neurogenic lineage. We further analyzed the role of inductive signals (NGF) on the differentiation of encapsulated MSCs. MSCs encapsulated in alginate/HA at a 1:1 w/w ratio without the presence of NGF exhibited very low levels of $\beta$ III-tubulin expression, while encapsulated MSCs in the presence of NGF expressed modest levels of $\beta$ III-tubulin expression (Figure 5d) confirming the importance of the presence of inductive signals on lineage differentiation of encapsulated MSC. The same trend in gene expression levels was obtained for hBMMSCs, PDLSCs, and GMSCs.

\subsection{In vivo 3D differentiation of encapsulated MSCs}

In vivo neurogenic differentiation of encapsulated PDLSCs and GMSCs was assessed four weeks after implantation via histochemical and Immunofluorescence staining. Encapsulated PDLSCs and GMSCs in HA/alginate hydrogel (1:1 w/w ratio) containing $\beta$-NGF were implanted subcutaneously in immunocompromised mice. hBMMSCs encapsulated in HA/ alginate hydrogel were used as the positive control and cell-free HA/alginate hydrogel was used as the negative one. Immunofluorescent staining was used to characterize the neurogenic differentiation of MSCs in vivo. Extensive staining with GFAP and $\beta$ III-tubulin antibodies was observed within the regenerated tissues 4 weeks after subcutaneous implantation (Figure 6a). We also investigated the expression of VAMP2, a synaptic protein. Our data showed positive staining in all the tested groups (Figure 6b). Our semi-quantitative analysis showed that engrafted PDLSCs had significantly higher expression levels of neurogenic-related genes compared to GMSCs $(\mathrm{p}<0.05)$ but not hBMMSCs $(\mathrm{p}>0.05)$ (Figure 6c). Furthermore, higher cell densities and greater numbers of cell colonies were observed for PDLSCs and hBMMSCs, while the negative control group failed to present any positive staining. 


\section{Discussion}

Despite all the recent advances in cell therapy, biomaterials science and tissue engineering, functional nerve regeneration is still a challenging clinical situation in regenerative medicine and often leads to disappointing outcomes. ${ }^{[49]}$ Cell therapies are considered a promising alternative treatment modality for nerve regenerations. In this process, progenitor stem cells will be engrafted at the defect site to promote cell differentiation toward the desired phenotype (neurogenic tissue) based on the presence of signaling molecules (e.g., neurogenic factors) and physiomechanical properties of the environment. ${ }^{[49]}$ Several studies have reported the application of different types of adult stem cells (e.g., hBMMSCs, adipose-derived stem cells, and dental pulp stem cells) for neurogenic differentiation. ${ }^{[50,51]}$ Dental and orofacial-derived MSCs (e.e. PDLSCs and GMSCs) are unique alternatives. These two categories of MSCs are easily available in the oral cavity; indeed, they can also be found in dental clinics tissue waste. ${ }^{[27-30]}$ However, no reports have assessed the neurogenic regenerative capability of PDLSCs and GMSCs encapsulated in RGD modified alginate/ hyaluronic acid hydrogels. In this study, for the first time, we investigated the suitability of PDLSCs and GMSCs delivered in this manner for nerve regeneration.

Our in vitro analysis showed that PDLSCs and GMSCs adopted the morphology of neuronal-like cells and expressed neurogenic-associated markers after two weeks of neurodifferentiation, as confirmed by positive $\beta$ III-tubulin immuno-staining. Interestingly, PDLSCs and GMSCs exhibited higher levels of $\beta$-tubulin III expression than hBMMSCs, a phenomenon that might be attributed to the neural crest origin of the orofacial-derived MSCs. Subsequently, we encapsulated PDLSCs and GMSCs in a 3D scaffold of alginate and hyaluronic acid (HA) hydrogel containing nerve growth factor, and analyzed the fate of MSCs via well-established in vitro and in vivo models. Studies have shown that a HA-rich scaffold with a low elastic modulus provides a favorable environment for neural differentiation. In addition, alginate hydrogel has been widely used in tissue engineering applications, including nerve regeneration. ${ }^{[40,41]}$ In this study, we first fabricated hydrogel scaffolds based on alginate and HA hydrogels with different composition and elasticity. We analyzed the influence of the biomechanical properties of the 3D hydrogels on proliferation and differentiation of encapsulated PDLSCs and GMSCs. As expected, a higher concentration of HA decreased the elastic modulus of the fabricated hydrogel mixture. Interestingly, we found that the proliferation rate of GMSCs increased with a decrease in the elasticity of the hydrogel, while scaffold elasticity did not influence the proliferation rates of PDLSCs or hBMMSCs. Additionally, neurogenic induction assays of the encapsulated cells showed that addition of HA to the alginate, in the presence of NGF, significantly enhanced the neurogenic differentiation of encapsulated MSCs, as confirmed by immunostaining and qPCR analyses. Immunostaining further revealed that MSCs encapsulated in the hydrogel with lowest elastic modulus showed the largest amount of $\beta$-tubulin III staining.

Encapsulating biomaterial plays a fundamental role in the successful in vivo application of MSCs in tissue engineering. ${ }^{[52,53]}$ Studies have confirmed that the porous structure of the scaffold can be advantageous for cell attachment, proliferation and differentiation, leading to the desired cell phenotype. ${ }^{[52-55]}$ An alginate/HA hydrogel system has an ideal porous structure, which supports cell adhesion and migration and permits transportation of oxygen, 
nutrients, waste molecules, and growth factors, influencing cell behavior and phenotype.

${ }^{[52-55]}$ In our previous studies, alginate hydrogel was utilized as a 3D scaffold for the encapsulation of MSCs during proliferation and differentiation. ${ }^{[28,29,56]}$ Furthermore, many studies have shown that HA hydrogel can support differentiation of embryonic and neural stem cells. ${ }^{[44-46]}$ Therefore, in the current study, HA was added to alginate to develop a dental-derived MSC delivery vehicle with neurogenic differentiation potential. Our results demonstrate that the addition of HA to the alginate hydrogel can enhance cell proliferation and neurogenic differentiation, probably due to the ability of HA to anchor to the cell surface via CD44 cell surface receptors. ${ }^{[57]}$ It has been reported that CD44 receptor is expressed in PDLSCs and GMSCs, and that it can bind HA. ${ }^{[58,59]}$ Our findings correlated well with previous reports that an alginate/HA scaffold better promotes both proliferation and neurogenic differentiation of encapsulated MSCs than alginate alone. The interaction of the biomaterial matrix and the encapsulated MSCs can be facilitated by the presence of HA and RGD tripeptide, enhancing cell adhesion and viability via increased access of cells to oxygen, nutrients, and growth factors. ${ }^{[28,29,57]}$

It has to be mentioned that not only addition of HA changes the elasticity of the hydrogel biomaterial, but also it might change the biochemical characteristics leading to enhanced differentiation of encapsulated MSC toward nerve-like tissues. In the current study, HA also was not utilized as a study group as the main focus of this study was on the application of alginate hydrogel as the encapsulating biomaterial for dental- and orofacial-derived MSCs.

In our in vivo study, immunofluorescence staining of tissues regenerated by implanted MSCs confirmed positive staining against $\beta$-III tubulin for PDLSCs and GMSCs. Moreover, PDLSCs showed significantly higher levels of neurogenic-related markers than GMSCs or hBMMSCs, while GMSCs exhibited comparable expression levels of neurogenic-specific markers to hBMMSCs. This finding is very promising as PDLSCs and GMSCs share an advantage over hBMMSCs due to their ready availability and favorable growth properties.

We confirmed that the physiochemical properties of an encapsulating biomaterial (e.g., elasticity and the presence of cell binding motifs) and the presentation of signaling ligands (e.g., NGF) regulate the viability, function, and differentiation and therefore the fate of the implanted MSCs, leading to enhanced tissue regeneration. Our current findings suggest that besides the elastic modulus of the encapsulating biomaterial, the biochemical microenvironment also affects the fate of the engrafted MSCs. Another important parameter that affects the fate of the encapsulated MSCs is the presence of inductive signals, which direct their differentiation toward the desired lineage.

\section{Conclusion}

Taken together, our studies demonstrate that human periodontal ligament and gingival tissues are promising sources of stem/progenitor cells for nerve tissue engineering applications. We showed that the physiomechanical properties of the 3D scaffold on which these cells were delivered significantly influenced the proliferation and differentiation of encapsulated MSCs toward neurogenic tissues. Encapsulated GMSCs and PDLSCs within an alginate/hyaluronic acid-based hydrogel scaffold with appropriate elasticity, in the 
presence of a suitable growth factor, possess potential to be used in repair/regeneration of neurogenic tissues. Our findings confirmed that our 3D injectable cell delivery scaffold based on alginate/hyaluronic acid comprises a promising modality of treatment for nerve tissue regeneration and repair.

\section{Experimental Section}

\section{MSC culture}

Young healthy male individuals undergoing third molar extractions were chosen for extraction of gingival and PDL tissues according to IRB approval. GMSCs and PDLSCs were isolated and cultured in regular MSC culture media. Human bone marrow (hBM) MSCs, purchased from Lonza (Lonza Inc. Walkersville, MD), were used as the control group in this study. Passage four cells were utilized in all experiments. ${ }^{[23,47]}$

\section{MSC encapsulation within hydrogel biomaterial}

PDLSCs, GMSCs or hBMMSCs were encapsulated in alginate/hyaluronic acid hydrogel at a density of $2 \times 10^{6}$ cells $/ \mathrm{mL}$ hydrogel. $5 \%$ RGD-coupled alginate hydrogel $(1.2 \mathrm{w} / \mathrm{v} \%)$ (NovaMatrix FMC Biopolymer, Norway) was used after being purified and partially oxidized (1\%). ${ }^{[28,29]}$ Next, a 5\% w/v solution of hyaluronic acid (Sigma-Aldrich) in PBS was filtered $(0.22 \mu \mathrm{m}$ sterile syringe filter, Millipore) and alginate powder was added. We fabricated three different hydrogel mixtures with different alginate/HA ratios (4:1, 2:1, and $1: 1 \mathrm{w} / \mathrm{w}$ ), and therefore, different elastic modulus values, and compared them to alginate hydrogel without HA as the control.

Recombinant human $\beta$-NGF Protein (R\&D Systems Minneapolis, MN) $(50 \mathrm{ng} / \mathrm{mL}$ in PBS) was added to the HA/alginate hydrogel mixture, sterile filtered $(0.22 \mu \mathrm{m}$ filter $)$ and freezedried under reduced pressure. Hydrogel microsphere fabrication was accomplished by adding the alginate/HA mixture dropwise into a $100 \mathrm{mM} \mathrm{CaCl}_{2}$ solution. The hydrogel microbeads formed during this process were incubated at $37^{\circ} \mathrm{C}$ for $45 \mathrm{~min}$ to complete ionic cross-linking and washed with non-supplemented DMEM (x3). PDLSCs, GMSCs, and hBMMSCs encapsulated in alginate were used as controls to analyze the effect of the presence of HA on cell viability, adhesion, and neurogenesis. Cell-free HA/alginate hydrogel was used as a negative control in this study.

\section{Measurement of viability and proliferation of encapsulated MSCs}

A live-dead assay (Calcein AM/ethidium bromide homodimer-1, Invitrogen) was utilized to analyze the viability of encapsulated MSCs up to two weeks after culturing in regular culture media. NIH ImageJ software (NIH, Bethesda, MD) was used to quantify the percentage of live cells. Additionally, the proliferation of the encapsulated MSCs was evaluated. Briefly, after one week of culturing in regular culture media, the alginate hydrogels were dissolved in a $15 \mathrm{mM}$ sodium citrate solution. Next, extracted cells were cultured in 96-well plates and DNA content was evaluated using CyQuant ${ }^{\circledR}$ cell proliferation assay. 


\section{Characterization of the alginate/HA hydrogels}

The biomechanical properties of the prepared hydrogels with different alginate/HA ratios were measured using an Instron mechanical testing machine (Norwood, PA) at a compression rate of $0.5 \mathrm{~mm} \cdot \mathrm{min}^{-1}$; and the elastic modulus (E) of each hydrogel was calculated. ${ }^{[48]}$ The morphology and pore sizes of the fabricated hydrogels were analyzed using scanning electron microscopy (SEM) (JEOL 5300, Peabody, MA). ${ }^{[28,29]}$ The release profile of the NGF was evaluated using an anti-human recombinant human $\beta$-NGF ELISA kit (Abcam). Briefly, alginate/HA microspheres containing h-NGF $(50 \mathrm{ng} / \mathrm{mL})$ were incubated in $20 \mathrm{~mL}$ PBS solution at $37^{\circ} \mathrm{C}$ for a period of up to two weeks. At different time intervals, the medium was collected and the amounts of released h-NGF were measured using the ELISA kit. The amount of NGF remaining inside the hydrogel mixture was measured via immunofluorescence staining using antibodies against h- $\beta$ NGF (Abcam).

Next, the swelling kinetics of the fabricated hydrogels was evaluated according to the methods already in the literature. ${ }^{[29]}$ Briefly, the prepared microbeads were dried, weighed, and immersed in distilled water at $37{ }^{\circ} \mathrm{C}$. At every 30 min time interval, the swollen samples were weighed and the swelling ratio (SR) was calculated.

\section{In vitro neurogenic differentiation assay}

A neurogenic differentiation assay was performed in 2D and 3D culture conditions. To induce neurogenic differentiation, PDLSCs, GMSCs, and hBMMSCs were cultured in regular culture media containing human $\beta$ NGF $(50 \mathrm{nM} / \mathrm{mL})$ for two weeks, while the culture medium was changed twice per week. After two weeks the neurogenic differentiation of the MSCs was analyzed immunofluorescently using antibodies against $\beta$ III tubulin and counterstaining with DAPI. MSC cultures without human $\beta$ NGF were utilized as the negative control.

Additionally, to analyze the role of the scaffold's mechanical properties and the role of inductive signals on the neuro-differentiation of encapsulated MSCs, PDLSCs, GMSCs, and hBMMSCs $\left(2 \times 10^{6}\right.$ cells) were encapsulated in $1 \mathrm{~mL}$ of HA/alginate microspheres with different alginate/HA ratios. The constructs were cultured in regular culture media containing human $\beta$ NGF ( $50 \mathrm{nM} / \mathrm{mL}$ ). Cell-free HA/alginate microspheres without any growth factor were used as the control group. Two weeks after neurogenic induction, the specimens were fixed with $4 \%$ PFA, and paraffin sections were made. Neurogenic differentiation was evaluated by immuno-staining using antibodies against GFAP and $\beta I I I-$ tubulin (Abcam) at $4{ }^{\circ} \mathrm{C}$ overnight, detected using Alexa fluor conjugated secondary antibody (1:200 dilution, Invitrogen), and counterstained with DAPI.

\section{Quantitative real-time PCR assay and Western blot analysis}

Encapsulated MSCs were recovered from hydrogel microspheres after four weeks of neurogenic induction and total RNA was isolated using Trizol reagent (Invitrogen). RNA was reverse-transcribed and single-stranded cDNA was synthesized using a Superscript III cDNA synthesis kit (Invitrogen). Relative gene expression was calculated using the $2^{-\Delta \Delta C t}$ method, with normalization to the $\mathrm{Ct}$ of the housekeeping gene GAPDH (glyceraldehyde 3phosphate dehydrogenase). Primer sequences are described in Table 1. 
In order to run Western blot analysis, after two weeks of neuro-induction, MSCs were recovered from hydrogels and lysed using protein extraction buffer (Bio-Rad, Irvine, CA). The extracted proteins were fractionated in $10 \%$ sodium dodecyl sulfate-polyacrylamide gels (PAGE) and electrophoretically transferred to a nitrocellulose membrane (Bio-Rad). Next, the membranes were incubated with antibodies against rabbit polyclonal glial fibrillary acidic protein (GFAP) and $\beta$ III Tubulin antibodies (Abcam). The membranes were reprobed with an antibody against the housekeeping gene $\beta$-actin (Abcam).

\section{Subcutaneous transplantation of encapsulated MSCs}

All of the surgical procedures were performed based on approved animal protocols. $4 \times 10^{6}$ PDLSCs, GMSCs, or hBMMSCs were encapsulated in HA/alginate microspheres (with optimized elasticity and alginate/HA 1:1 ratio) loaded with $\beta$-NGF. Approximately 10 microspheres were implanted in each subcutaneous pocket into the dorsal surfaces of mice (5-month-old Beige nude XID III (NU/NU), Harlan, Livermore, CA). Seven mice were tested in each group. Each animal had four different implants: 1) PDLSCs, 2) GMSCs, 3) hBMMSCs (positive control) and 4) Cell-free hydrogel or MSC-hydrogel constructs without human $\beta$ NGF were utilized as the negative control. Four weeks after implantation, the mice were sacrificed, and the microspheres were harvested and stored for further analysis.

\section{Histological, histochemical and Immunofluorescence staining}

Four weeks post-implantation, the tissues were harvested, fixed in PFA (4\%) solution, embedded in paraffin, sectioned $(6 \mu \mathrm{m})$, and deparaffinized for staining. In order to identify neuron-like tissue formation, the sections were stained with primary antibodies monoclonal mouse anti- GFAP, anti- $\beta$ III-tubulin and anti-VAMP-2 antibodies (1:1000, Abcam). For immunofluorescence staining, cells were treated with $3 \% \mathrm{H}_{2} \mathrm{O}_{2}$ and then with a blocking buffer (1\% BSA and $0.25 \%$ Triton X-100 in PBS). Specimens were washed again with PBS and incubated with either anti- GFAP or anti- $\beta$-III tubulin primary antibody overnight. Subsequently, the specimens were incubated with secondary Alexa-Fluor conjugated goat anti-mouse IgG (1:200, Invitrogen) and counterstained with DAPI (Vector Laboratories, Burlingame, CA).

\section{Statistical Analysis}

Kolmogorov-Smirnov (K-S) (Normality test) test was performed (using SPSS software) to determine whether our obtained data were normally distributed or not. Based on the results of our normality test, the Kruskal-Wallis rank sum test or one-way ANOVA were used to analyze the data at a significance level of $a=0.05$. Also, two-tailed Student's t-tests were utilized for pairwise comparisons whenever needed. Quantitative data are expressed as mean \pm standard deviation $(\mathrm{SD})$.

\section{Supplementary Material}

Refer to Web version on PubMed Central for supplementary material. 


\section{Acknowledgments}

This work was supported by grants from the National Institute of Dental, Craniofacial Research (K08DE023825 to A.M.).

\section{References}

1. Evans GR. Semin Surg Oncol. 2000; 19:312. [PubMed: 11135488]

2. Fu SY, Gordon T. Mol Neurobiol. 1997; 14:67. [PubMed: 9170101]

3. Turnbull VJ. Methods Mol Med. 2005; 107:173. [PubMed: 15492372]

4. Amoh Y, Kanoh M, Niiyama S, Hamada Y, Kawahara K, Sato Y, Hoffman RM, Katsuoka K. J Cell Biochem. 2009; 107:1016. [PubMed: 19507228]

5. Tohill M, Mantovani C, Wiberg M, Terenghi G. Neurosci Lett. 2004; 362:200. [PubMed: 15158014]

6. Walsh SK, Gordon T, Addas BM, Kemp SW, Midha R. Exp Neurol. 2010; 223:221. [PubMed: 19477174]

7. Cui L, Jiang J, Wei L, Zhou X, Fraser JL, Snider BJ, Yu SP. Stem Cells. 2008; 26:1356. [PubMed: 18308951]

8. Guan K, Chang H, Rolletschek A, Wobus AM. Cell Tissue Res. 2001; 305:171. [PubMed: 11545254]

9. Gritti A, Parati EA, Cova L, Frolichsthal P, Galli R, Wanke E, Faravelli L, Morassutti DJ, Roisen F, Nickel DD, Vescovi AL. J Neurosci. 1996; 16:1091. [PubMed: 8558238]

10. Palmer TD, Ray J, Gage FH. Mol Cell Neurosci. 1995; 6:474. [PubMed: 8581317]

11. Reynolds BA, Weiss S. Science. 1992; 255:1707. [PubMed: 1553558]

12. Woodbury D, Schwarz EJ, Prockop DJ, Black IB. J Neurosci Res. 2000; 61:364. [PubMed: 10931522]

13. Dominici M, Le Blanc K, Mueller I, Slaper-Cortenbach I, Marini F, Krause D, Deans R, Keating A, Prockop DJ, Horwitz E. Cytotherapy. 2006; 8:315. [PubMed: 16923606]

14. Friedenstein AJ, Chailakhyan RK, Latsinik NV, Panasyuk AF, Keiliss-Borok IV. Transplantation. 1974; 17:331. [PubMed: 4150881]

15. Becker AJ, McCulloch EA, Till JE. Nature. 1963; 197:452. [PubMed: 13970094]

16. Chamberlain G, Fox J, Ashton B, Middleton J. Stem Cells. 2007; 25:2739. [PubMed: 17656645]

17. Bi Y, Ehirchiou D, Kilts TM, Inkson CA, Embree MC, Sonoyama W, Li L, Leet Al, Seo BM, Zhang L, Shi S, Young MF. Nature Medicine. 2007; 13:1219.

18. Romero-Ramos M, Vouch P, Young HE, Lucas PA, Wu Y, Chivatakam O, Zaman R, Dunkelman N, el-Kalay MA, Chesselet MF. J Neurosci Res. 2002; 69:894. [PubMed: 12205682]

19. Oliveira JT, Mostacada K, de Lima S, Martinez AM. Int Rev Neurobiol. 2013; 108:59. [PubMed: 24083431]

20. Ladak A, Olson J, Tredget EE, Gordon T. Exp Neurol. 2011; 228:242. [PubMed: 21281630]

21. Gronthos S, Brahim J, Li W, Fisher LW, Cherman N, Boyde A, DenBesten P, Robey PG, Sei S. J Dent Res. 2002; 81:531. [PubMed: 12147742]

22. Miura M, Gronthos S, Zhao M, Lu B, Fisher LW, Robey PG, Shi S. Proc Natl Acad Sci U S A. 2003; 100:5807. [PubMed: 12716973]

23. Seo B-M, Miura M, Gronthos S, Bartold PM, Batouli S, Brahim J, Young M, Robey PG, Wang CY, Shi S. Lancet. 2004; 364:149. [PubMed: 15246727]

24. Sonoyama W, Liu Y, Fang D, Yamaza T, Seo BM, Zhang C, Liu H, Growths S, Wang CY, Wang S, Shi S. PLoS One. 2006; 1:70.

25. Sonoyama W, Liu Y, Yamaza T, Tuan RS, Wang S, Shi S, Huang GT. J Endod. 2008; 34:166. [PubMed: 18215674]

26. Zhang Q, Shi S, Liu Y, Uyanne J, Shi Y, Shi S, Le AD. J Immunol. 2009; 183:7787. [PubMed: 19923445]

27. Moshaverinia A, Chen C, Xu X, Akiyama K, Ansari S, Zadeh HH, Shi S. Tissue Eng Part A. 2014; 20:611. [PubMed: 24070211] 
28. Moshaverinia A, Chen C, Akiyama K, Xu X, Chee WW, Schricker SR, Shi S. J Biomed Mater Res A. 2013; 101:3285. [PubMed: 23983201]

29. Moshaverinia A, Chen C, Akiyama K, Ansari S, Xu X, Chee WW, Schricker SR, Shi S. J Mater Sci Mater Med. 2012; 23:3041. [PubMed: 22945383]

30. Moshaverinia A, Xu X, Chen C, Akiyama K, Snead ML, Shi S. Acta Biomater. 2013; 9:9343. [PubMed: 23891740]

31. Moshaverinia A, Ansari S, Chen C, Xu X, Akiyama K, Snead ML, Zadeh HH, Shi S. Biomaterials. 2013; 34:6572. [PubMed: 23773817]

32. Moshaverinia A, Chen C, Xu X, Ansari S, Zadeh HH, Schricker SR, Paine ML, Moradian-Oldak J, Khademhosseini A, Snead ML, Shi S. Adv Funct Mater. 2015; 25:2296. [PubMed: 26120294]

33. Lee KY, Rowley JA, Eiselt P, Moy EM, Bouhadir KH, Mooney DJ. Macromolecules. 2000; 33:4291.

34. Strand B, Gåserød O, Kulseng B, Espevik T, Skjåk-Bræk G. J Microencapsul. 2002; 19:615. [PubMed: 12433304]

35. Banerjee A, Arha M, Choudhary S, Ashton RS, Bhatia SR, Schaffer DV, Kane RS. Biomaterials. 2009; 30:4695. [PubMed: 19539367]

36. Lee KY, Mooney DJ. Prog Polym Sci. 2012; 37:106. [PubMed: 22125349]

37. Boontheekul T, Kong H-J, Mooney DJ. Biomaterials. 2005; 26:2455. [PubMed: 15585248]

38. Bouhadir KH, Lee KY, Alsberg E, Damm KL, Anderson KW, Mooney DJ. Biotechnol Prog. 2001; 17:945. [PubMed: 11587588]

39. Drury JL, Mooney DJ. Biomaterials. 2003; 24:4337. [PubMed: 12922147]

40. Bozza A, Coates EE, Incitti T, Ferlin KM, Messina A, Menna E, Bozzi Y, Fisher JP, Casarosa S. Biomaterials. 2014; 35:4636. [PubMed: 24631250]

41. Li L, Davidovich AE, Schloss JM, Chippada U, Schloss RR, Langrana NA, Yarmush ML. Biomaterials. 2011; 32:4489. [PubMed: 21481927]

42. Kang SW, Cha BH, Park H, Park KS, Lee KY, Lee SH. Macromol Biosci. 2011; 11:673. [PubMed: 21337520]

43. Re'em T, Tsur-Gang O, Cohen S. Biomaterials. 2010; 31:6746. [PubMed: 20542332]

44. Margolis RU, Margolis RK, Chang LB, Preti C. Biochemistry. 1975; 14:85. [PubMed: 122810]

45. Preston M, Sherman LS. Front Biosci. 2011; 3:1165.

46. Bandtlow CE, Zimmermann DR. Physiol Rev. 2000; 80:1267. [PubMed: 11015614]

47. Xu X, Chen C, Akiyama K, Chai Y, Le AD, Wang Z, Shi S. Gingivae contain neural-crest- and mesoderm-derived mesenchymal stem cells. J Dent Res. 2013; 92:825. [PubMed: 23867762]

48. Chaudhuri O, Gu L, Klumpers D, Darnell M, Bencherif SA, Weaver JC, Huebsch N, Lee HP, Lippens E, Duda GN, Mooney DJ. Nat Mater. 2016; 15:326. [PubMed: 26618884]

49. Lavasani M, Thompson SD, Pollett JB, Usas A, Lu A, Stolz DB, Clark KA, Sun B, Péault B, Huard J. J Clin Invest. 2014; 124:1745. [PubMed: 24642464]

50. Karbanová J, Soukup T, Suchánek J, Pytlík R, Corbeil D, Mokrý J. Cells Tissues Organs. 2011; 193:344. [PubMed: 21071916]

51. Kiraly M, Porcsalmy B, Pataki A, Kádár K, Jelitai M, Molnár B, Hermann P, Gera I, Grimm WD, Ganss B, Zsembery A, Varga G. Neurochem Int. 2009; 55:323. [PubMed: 19576521]

52. Liu J, Sato C, Cerletti M, Wagers A. Curr Top Dev Biol. 2010; 92:367. [PubMed: 20816402]

53. Kratchmarova I, Blagoev B, Haack-Sorensen M, Kassem M, Mann M. Science. 2005; 308:1472. [PubMed: 15933201]

54. Cantu DA, Hematti P, Kao WJ. Stem Cells Transl Med. 2012; 1:740. [PubMed: 23197666]

55. Ansari S, Moshaverinia A, Han A, Pi SP, Abdelhamid AI, Zadeh HH. Biomaterials. 2013; 34:0191.

56. Ansari S, Chen C, Xu X, Annabi N, Zadeh HH, Wu BM, Khademhosseinin A, Shi S, Moshaverinia A. Annals Biomed Eng. 2016; 40:1908.

57. Knudson CB. Birth Defects Res C Embryo Today. 2003; 69:174. [PubMed: 12955860]

58. Iwasaki K, Komaki M, Yokoyama N, Tanaka Y, Taki A, Honda I, Kimura Y, Takeda M, Akazawa K, Oda S, Izumi Y, Morita I. Tissue Eng Part A. 2014; 20:693. [PubMed: 24032400] 
59. Mitrano TI, Grob MS, Carrión F, Nova-Lamperti E, Luz PA, Fierro FS, Quintero A, Chaparro A, Sanz A. J Periodontol. 2010; 81:917. [PubMed: 20450355]

60. Kutty JK, Cho EH, Lee JS, Vyavahare NR, Webb K. Biomaterials. 2007; 28:928.

61. Gurski L, Petrelli N, Jia X, Farach-Carson M. Oncol Issues. 2010; 25:20. 

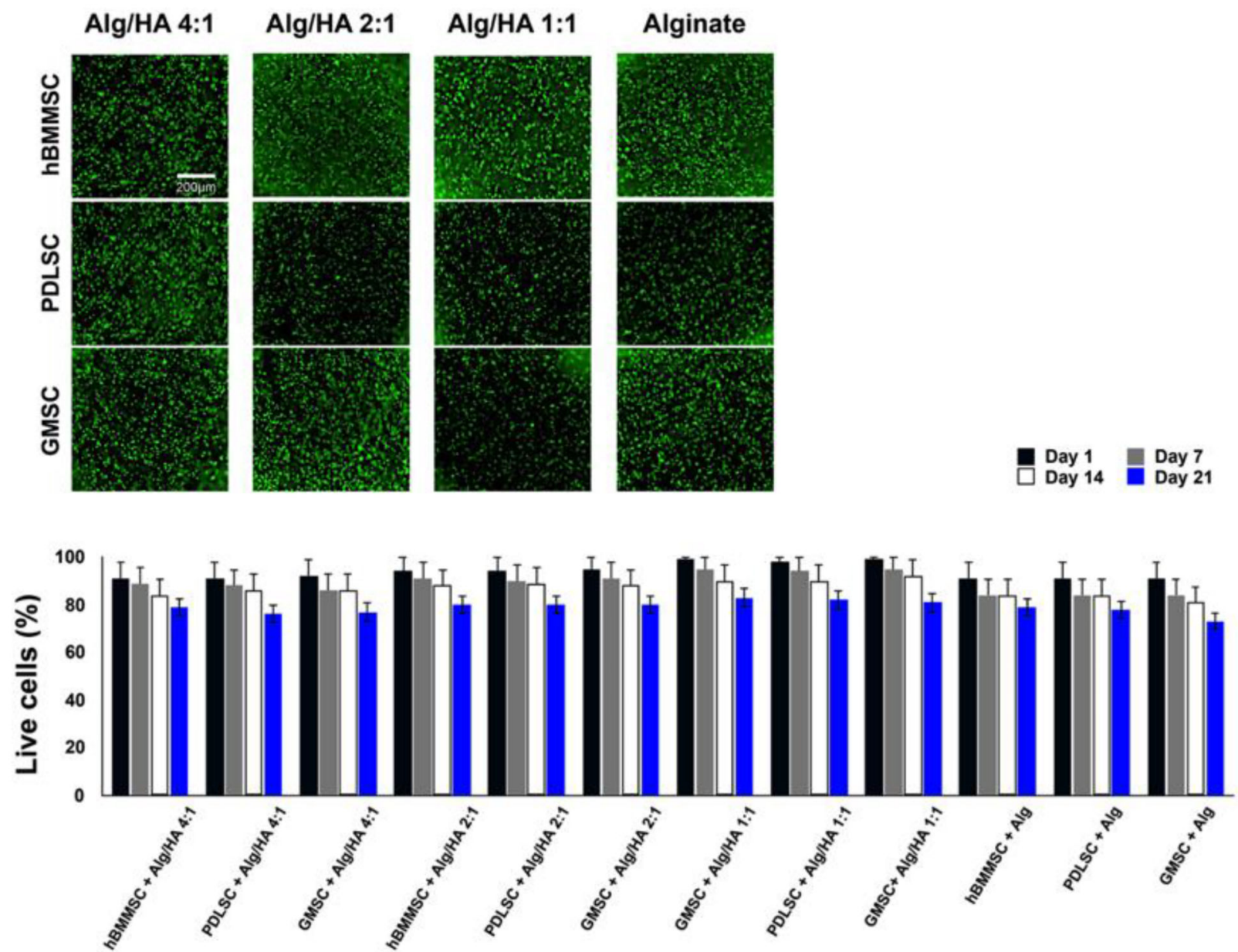

Figure 1.

The viability of the encapsulated PDLSCs, GMSCs, and hBMMSCs: (a) Live/dead staining of hBMMSCs, GMSCs and PDLSCs encapsulated in alginate/HA hydrogel microspheres after one week of culturing in regular culture media. (b) Percentage of live cells measured at different time points, indicating that more than $79 \%$ of encapsulated MSCs remained viable for three weeks. 
a

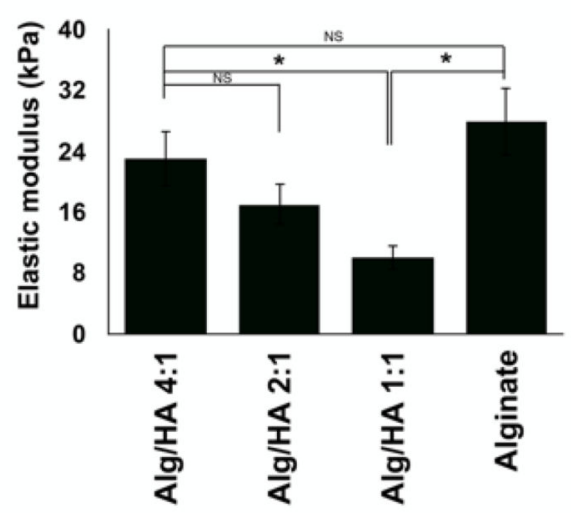

b
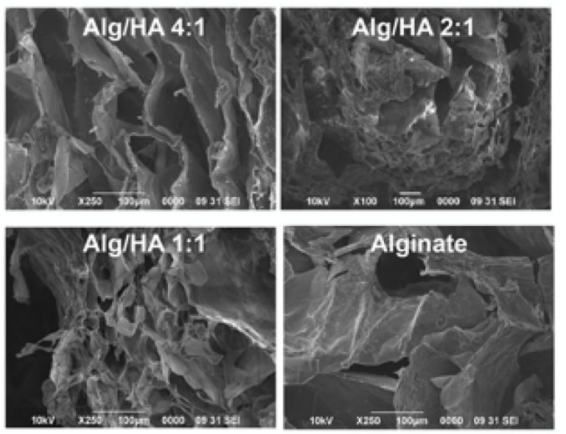

- Day 1

d

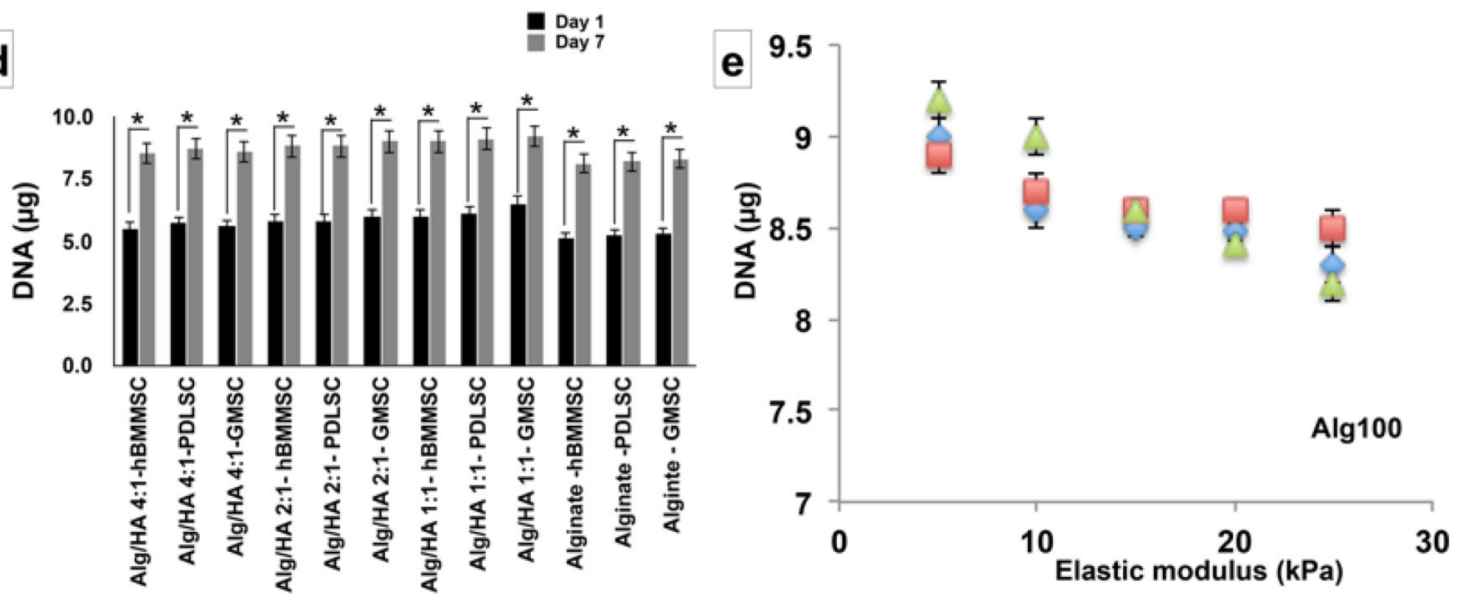

${ }_{\text {hBMMSC }}$

PDLSC

$\triangle$ GMSC

Alg200

Figure 2.

Characterization of fabricated hydrogel microspheres and proliferation of encapsulated MSCs: (a) Elastic modulus of the fabricated hydrogel microspheres with different Alg/HA ratios. (b) SEM image of fabricated alginate/HA hydrogels confirming their micro-porous structure of the hydrogel matrix. (c) The average pore size for the fabricated hydrogels based on SEM images. (d) Influence of composition of the fabricated hydrogel on the proliferation of encapsulated MSCs. (e) The relationship between the elastic modulus of the encapsulating biomaterial (alginate/HA 1:1), type of MSC, and the proliferation rate of each type of cell. Six independent specimens per group were analyzed in each study. ${ }^{*} \mathrm{p}<0.05$. 
a

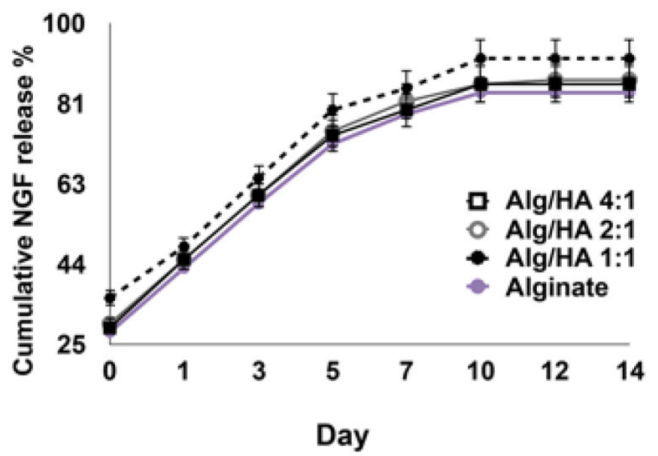

b

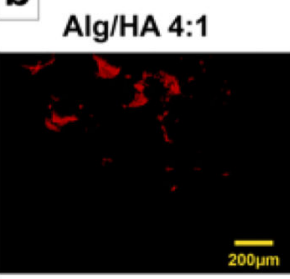

Alg/HA 1:1
Alg/HA 2:1

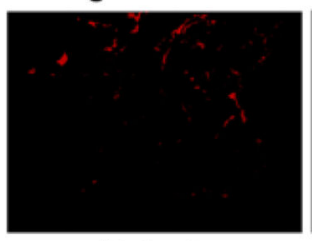

Alginate

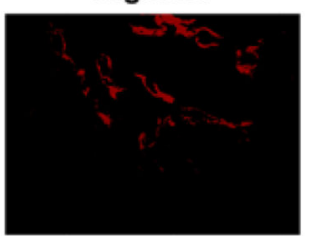

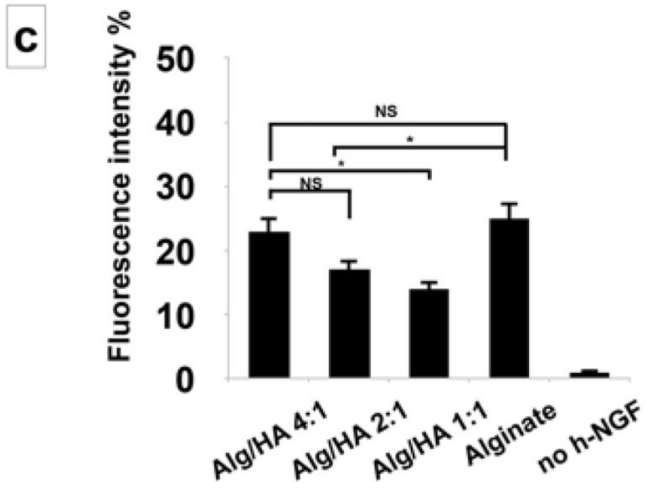

d

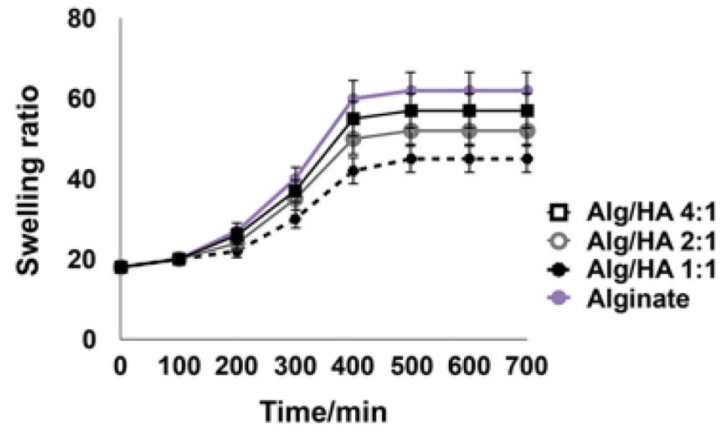

Figure 3.

Release profile characterization: (a) In vitro release profile characterization of human $\beta$ -

NGF from alginate/HA hydrogels, confirming sustained release of $\beta$-NGF. A slight increase in the initial release of the growth factor was observed from the softer hydrogel mixture with the higher concentration of HA; however, no significant difference was observed in release rates of growth factors from the different scaffolds $(p>0.05)$, and both exhibited first-order kinetics. (b) Immunofluorescence staining against anti- $\beta$-NGF antibodies showing the retained NGF ligands remained within alginate/HA with 4:1, 2:1, 1:1 ratios and alginate alone after 14 days. (c) Semi-quantitative analysis of retained NGF ligands in panel b. (d) Swelling properties of the fabricated hydrogels and the effects of the HA ratio. Six independent specimens per group were analyzed in each study. $* P<0.05$, NS: not significant. 

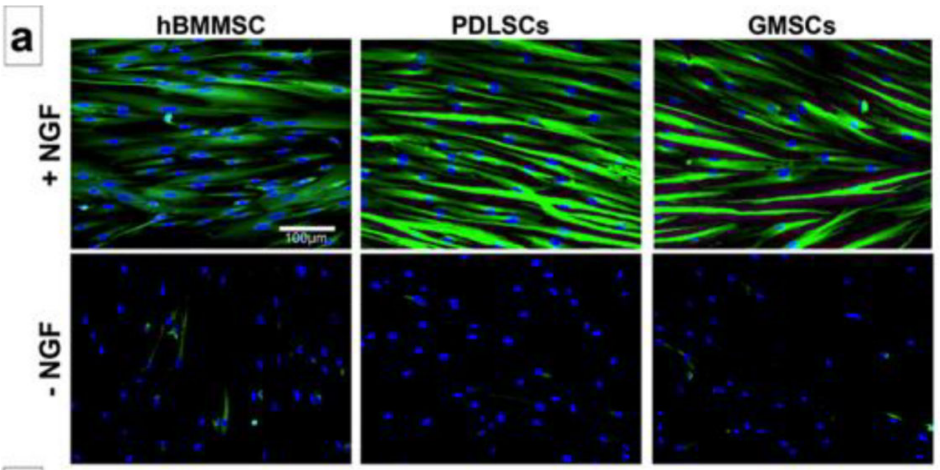

b
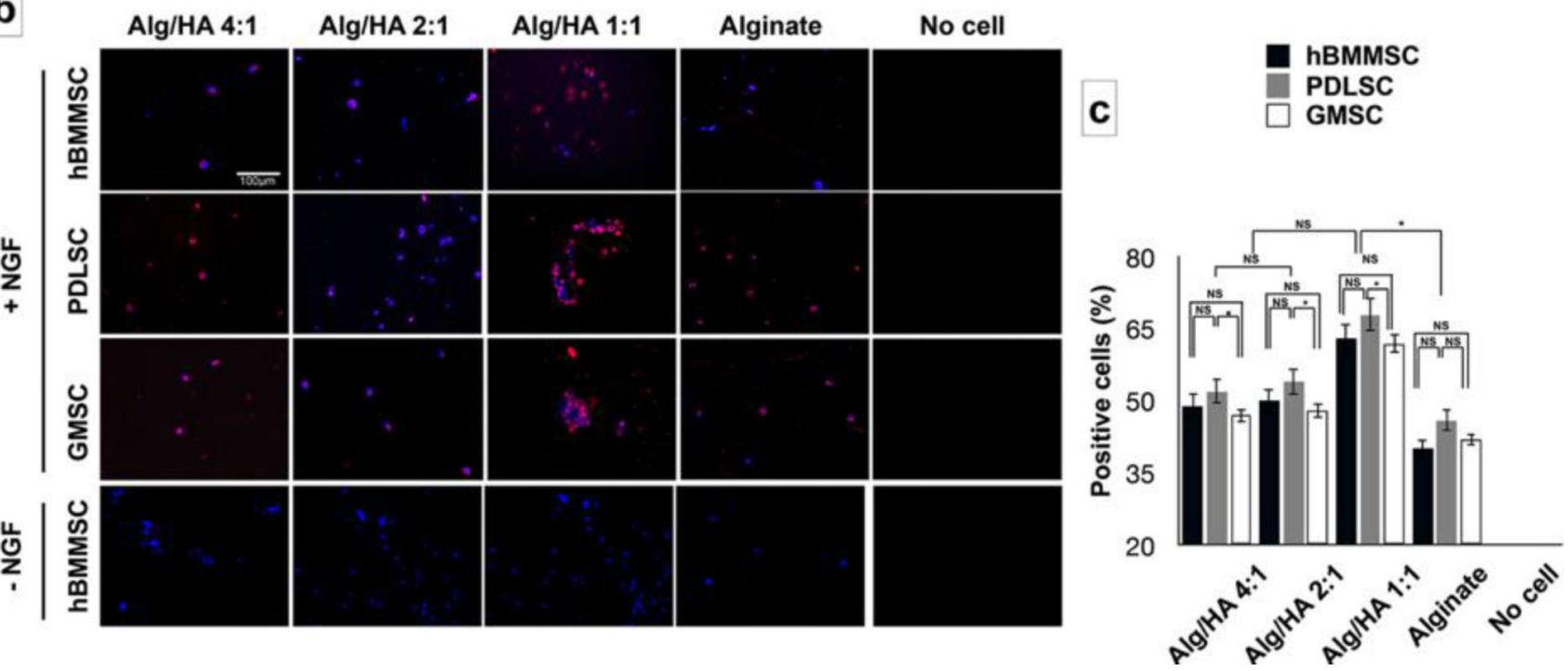

Figure 4.

In vitro neurogenic differentiation of encapsulated MSCs. (a) Immunofluorescence staining against $\beta$-tubulin III antibodies for hBMMSCs, PDLSCs, and GMSCs after two weeks of neurogenic induction assay. The effects of presence or absence of beta NGF was analyzed. (b) Immunofluorescence staining against $\beta$-tubulin III antibodies for encapsulated MSCs against $\beta$-tubulin III antibodies showing positively staining for all the tested MSCs in different hydrogel scaffolds. hBMMSCs encapsulated in hydrogel without NGF was utilized as the control and failed to express any positive staining. (c) The percentage of positive stained cells (from panel b) for MSCs encapsulated in hydrogel microspheres. Six independent specimens per group were analyzed in each study. ${ }^{*} \mathrm{P}<0.05, * * \mathrm{P}<0.01$, NS: not significant. 
a
$\beta$ tubulin III
Alg/HA 4:1
$\mathrm{Alg} / \mathrm{HA} 1: 1$
Alginate

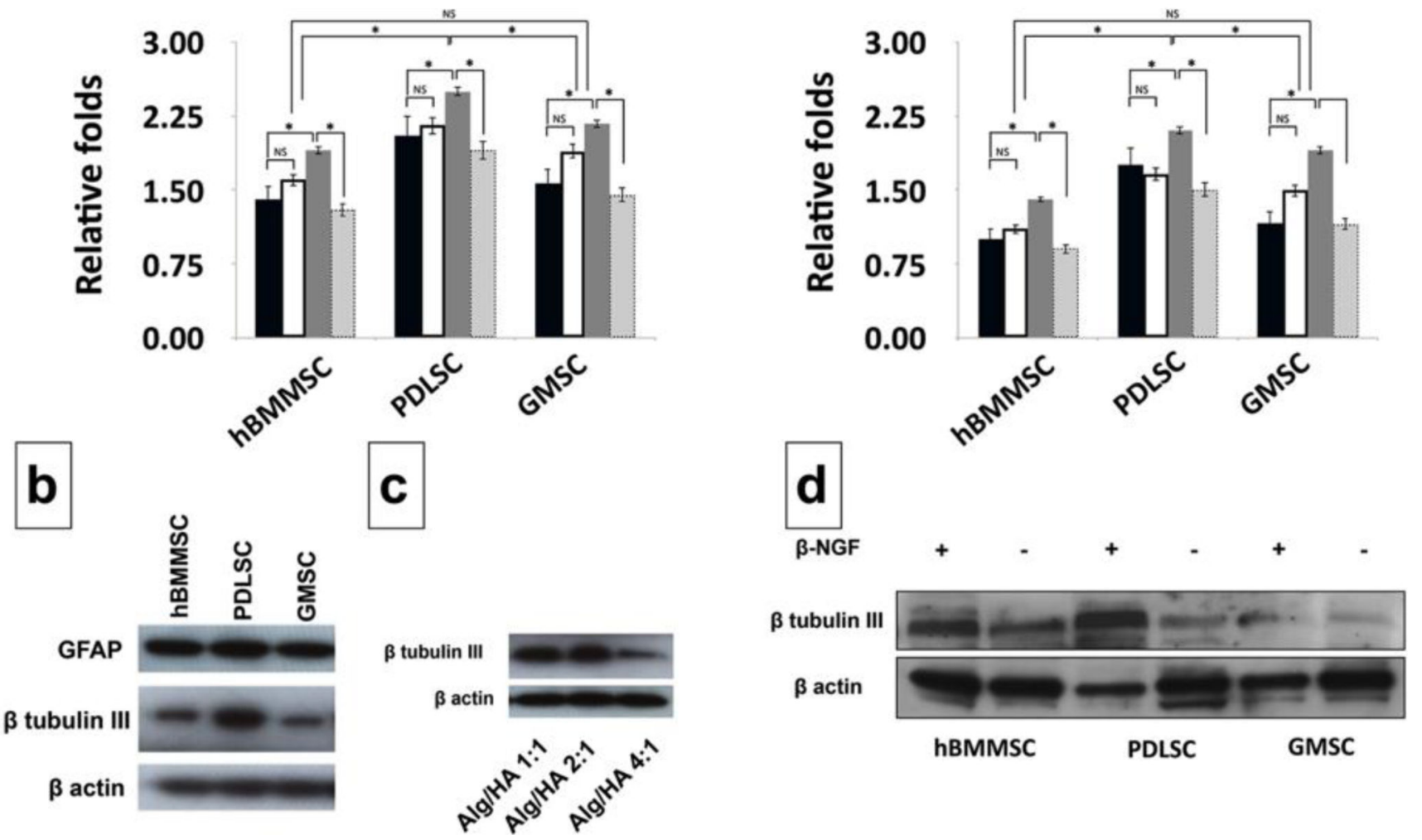

Figure 5.

Molecular analysis of neuro-differentiation of encapsulated MSCs. (a) The expression levels of $\beta$-tubulin III and glial fibrillary acidic protein (GFAP) genes after 14 days of neurogenic induction in vitro, analyzed using RT-PCR. (b) Western blot analysis exhibiting the expression levels of neurogenic regulator gene GFAP and $\beta$-tubulin III in MSCs encapsulated in Alg/HA 1:1 hydrogel mixture. The levels of GFAP and $\beta$-tubulin III expression were higher for PDLSCs than for hBMMSCs and GMSCs. (c) Expression levels of $\beta$-tubulin III for encapsulated PDLSCs confirming that application of the hydrogel biomaterial with lower elastic modulus led to enhanced $\beta$-tubulin III expression levels (d) Presence of inductive signal (NGF) led to greater $\beta$-tubulin III expression levels for encapsulated MSCs in alginate/HA hydrogel with 1:1 w/w ratio. Six independent specimens per group were analyzed in each study. $* P<0.05$, NS: not significant. 
a
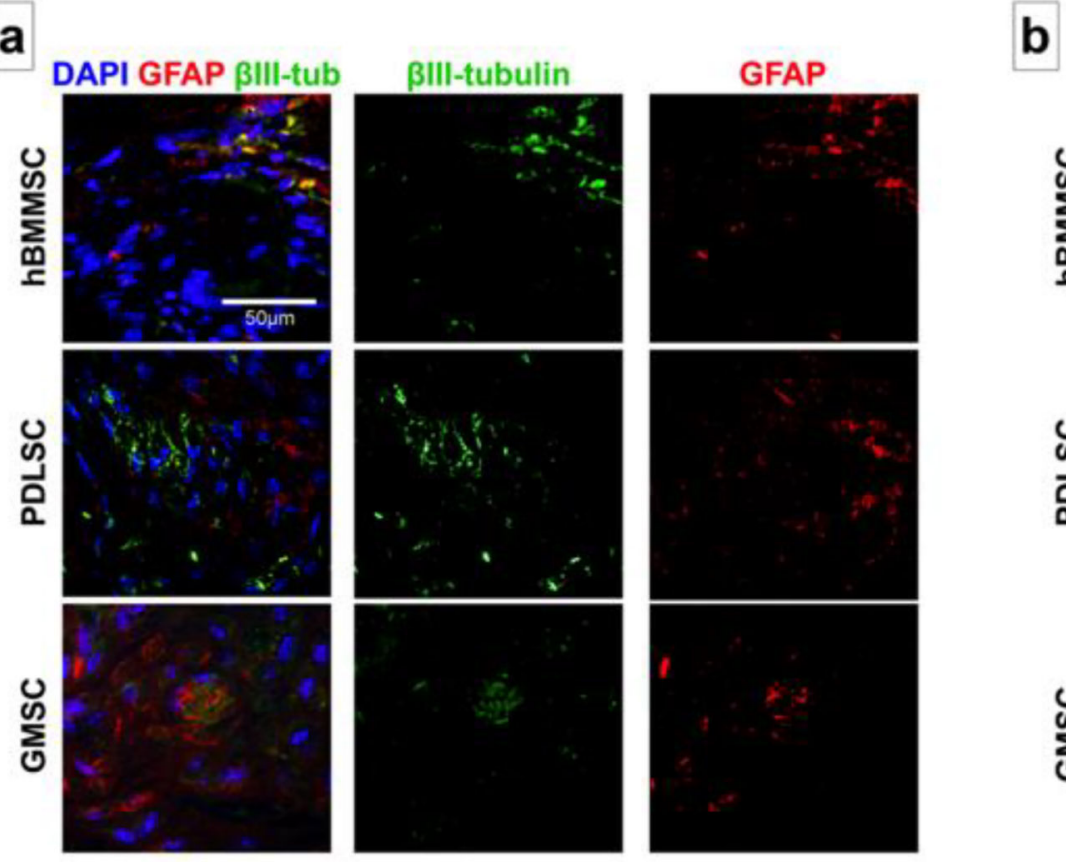

b
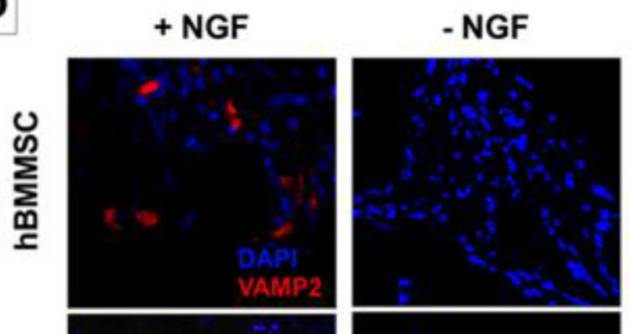

C

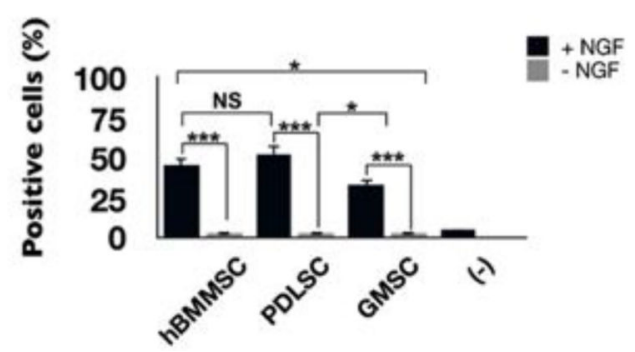

Figure 6.

Subcutaneous transplantation of encapsulated MSCs in HA/alginate hydrogel with 1:1 w/w ratio: (a) Immunofluorescence staining for early neural differentiation markers GFAP (red) and $\beta$ III-tubulin (green); and (b) pre-synaptic marker VAMP2 and the effects of presence of beta NGF. (c) The percentage of positive cells for VAMP2 antibody in each specimen according to immunostaining results of panel $b .{ }^{*} P<0.05$, NS: not significant.

Adv Healthc Mater. Author manuscript; available in PMC 2018 December 01. 


\section{Table 1}

Oligonucleotide primers used in RT-PCR analysis.

\begin{tabular}{l|l|l|}
\hline Gene & Sequence & $\begin{array}{l}\text { Amplification } \\
\text { (bp) }\end{array}$ \\
\hline GFAP & $\begin{array}{l}\text { Sense: 5'-GGAGAGGGGACAACTTTGCAC -3' } \\
\text { Antisense: 5'-CCAGCGATTCAACCTTTCTC -3' }\end{array}$ & 164 \\
\hline$\beta$-tubulin III & Sense: 5'- TTCTGGTGGACTTGGAACCT-3' & 180 \\
& Antisense: 5'- ACTCTTTCCGCACGACATCT-3' & \\
\hline Glyceraldehyde 3-phosphate dehydrogenase (GADPH) & Sense: 5'-AGCCGCATCTTCTTTTGCGTC-3': & 418 \\
& Antisense: 5'-TCATATTTGGCAGGTTTTTCT-3' & \\
\hline
\end{tabular}

